\title{
Declination Dependence of the Telescope Array Surface Detector Spectrum
}

\author{
Dmitri Ivanov ${ }^{* \dagger}$ \\ University of Utah, Department of Physics \& Astronomy and High Energy Astrophysics Institute, \\ Salt Lake City, Utah, USA \\ E-mail: dmiivanov@gmail.com
}

\begin{abstract}
The cosmic ray spectrum at ultra high energies $(\mathrm{E}>1 \mathrm{EeV})$ has two features: the ankle near $3 \mathrm{EeV}$ and the so-called second break point, at $60 \mathrm{EeV}$. If cosmic rays were pure protons at the highest energies, the second break point is explained by the well known Greissen-ZatsepinKuz'min process: energy loss of the protons on cosmic microwave background (CMB) due to the photo-pion production. In the case of a mixed chemical composition, the prediction for the position of the second break point is complicated by the spallation of the heavier nuclei on the cosmic microwave background. In the second case, the energy of the second break point could be lower than that of pure protons. Both Telescope Array and Pierre Auger experiments have measured the spectrum at the highest energies, and the results are in good agreement from 0.1 to $25 \mathrm{EeV}$ when the two measurements are adjusted to use a common energy scale. Above 25 $\mathrm{EeV}$, however, there is a significant discrepancy between the two results: the second break point in Pierre Auger spectrum occurs at a significantly lower energy than that of the Telescope Array. This effect cannot be explained by adjusting the energy scales of the two experiments.

In this work, we use data of the Telescope Array surface detector to show evidence of the dependence of the second break point energy on the declination. When we restrict the TA declination to a range from -15 to $24.8^{\circ}$, we see that the second break point occurs at a lower energy of $40 \mathrm{EeV}$, in better agreement with the Pierre Auger result. The difference between the TA low and high declination break points is a $3.9 \sigma$ effect. Also, we perform checks of the systematic uncertainties and demonstrate that this is not an instrumental effect.
\end{abstract}

35th International Cosmic Ray Conference - ICRC2017

10-20 July, 2017

Bexco, Busan, Korea

* Speaker.

${ }^{\dagger}$ For the Telescope Array Collaboration 


\section{Introduction}

Ultra-high energy cosmic ray energy spectrum has been measured accurately by three recent experiments: High Resolution Fly's Eye (HiRes) [1], Pierre Auger Observatory (Auger) [2], and the Telescope Array (TA) [3]. Although these experiments have vastly different exposures and use generally different detection techniques, all three agree that the cosmic ray spectrum at ultrahigh energies has an ankle feature near $3 \mathrm{EeV}$ and the second break point at $60 \mathrm{EeV}$. In the case of HiRes and TA, the ankle feature is explained by the electron-positron pair production on the cosmic microwave background (CMB) [4] and the second break point is explained by the photo-pion production on the $\mathrm{CMB}$, also known as the GZK process [5, 6]. This interpretation is consistent with the results of the mass composition measurements by HiRes [7] and TA [8], which report a light mass composition at the ultra-high energies. In the case of Auger, on the other hand, the effects of the propagation are complicated by the mixed composition result that is reported by the Auger experiment [9] and by the fact that the second break point in the Auger spectrum occurs at a lower energy of about $40 \mathrm{EeV}$ [2].

If the declination range of the TA SD spectrum measurement is restricted to the range of the sensitivity of the Auger SD analysis, from -15 to $24.8^{\circ}$, the second break point of the TA SD spectrum moves from $60 \mathrm{EeV}$ to $40 \mathrm{EeV}$, and the TA spectrum is in a better agreement with the Auger spectrum. The goal of this work is to present the result and to study the related systematic uncertainties.

\section{TA Surface Detector Data}

The Telescope Array (TA) is a cosmic ray detector in the Northern hemisphere initially designed to measure ultra high energy cosmic rays above $1 \mathrm{EeV}$. The sensitivity of TA has been recently extended to $4 \mathrm{PeV}$ with the TA low energy extension (TALE). Figure 1 shows the configuration of the entire TA detector using a Cartesian coordinate system that is centered at the TA Central Laser Facility (CLF). The main TA detector consists of the three fluorescence detector (FD) stations: Black Rock Mesa, Long Ridge [10], and Middle Drum [11], that are overlooking a surface detector array of 507 counters [12]. Each TA SD counter consists of 2 layers of $3 \mathrm{~m}^{2} \times 1.2 \mathrm{~cm}$ plastic scintillator and uses a $50 \mathrm{MHz} 12$ bit FADC readout. The counters are deployed on a $1200 \mathrm{~m}$ square grid so that the TA SD effectively covers a $680 \mathrm{~m}^{2}$ area on the ground. In this work, we use the data collected by the TA SD in a period from May 11, 2008 to May 11, 2015.

The TA SD counters are powered by the solar cells and are calibrated using the atmospheric muons every 10 minutes. The purpose of the calibration is to determine the size of the signal that corresponds to the energy deposition of a minimum ionizing particle (MIP) and the vertical equivalent muon (VEM). The TA SD uses radio readout system and triggers on events when 3 adjacent detectors report pulse heights exceeding 3 MIPs within an 8mus window. When the event trigger occurs, all counters that fired with pulse heights exceeding $0.3 \mathrm{MIP}$ in a $\pm 32 \mu$ s time interval send their waveforms to the data acquisition communication tower. TA SD event reconstruction consists of two parts: a time fit to determine the geometry of the shower track, and a fit to the lateral distribution of the counter pulse heights to determine the quantity called 5800 , which is the energy deposition per unit area at an 800m distance from the shower axis [13]. The energy of 


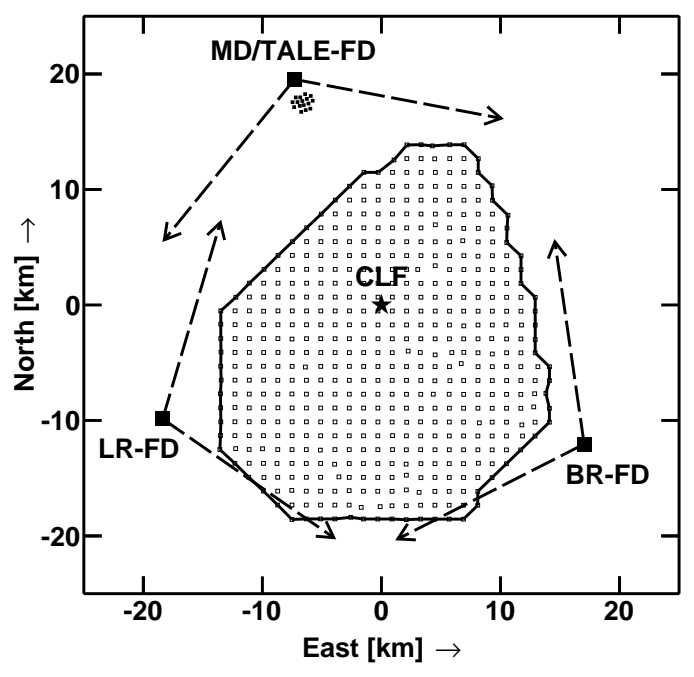

Figure 1: Layout of the Telescope Array detector. Filled star represents the position of the TA Central Laser Facility (CLF). Open squares show the locations of the main TA SD counters, small filled squares correspond to the TALE infill array counters, and the large squares with arrows show the positions and the fields of view of the three TA FD sites: Black Rock Mesa (BR), Long Ridge (LR), and Middle Drum (MD).

the primary particle is inferred from the energy estimation table, which maps $\mathrm{S} 800, \sec (\theta)$ to the energy of the cosmic ray ( $\theta$ is the shower zenith angle). The energy estimation table is made from a detailed Monte Carlo (MC) that uses CORSIKA [14] with QGSJETII.3 [15]. In order to produce large Monte Carlo sets, a thinning [16] approximation is used in the CORSIKA simulations of the showers above $0.1 \mathrm{EeV}$. We then restore the lost shower information on the ground by a dethinning technique [17]. Finally, we calibrate the energies obtained from the MC energy estimation table to the FD energy scale because the FD provides a calorimetric energy determination that is more accurate. The calibration of the SD energy scale is done by comparing the events that have been seen by the SD and FD in common. In order to match the energies of the FD, the SD energies obtained from the MC energy estimation table need to be scaled down by a constant factor of 1.27 [3], which is independent of energy, as shown in section 4. Above $10 \mathrm{EeV}$, the TA SD has a $1.5^{\circ}$ angular resolution and an energy resolution better than $20 \%$ [13].

\section{Results}

Figure 2 shows the results of the TA and Auger full sky energy spectra superimposed on top of each other, where the Auger energy has been scaled up by 1.16 to match the energy scale of the TA. The two results agree very well below $25 \mathrm{EeV}$. After $25 \mathrm{EeV}$, there is a very large discrepancy between the Auger and TA results. We see a better agreement of the TA energy spectrum with the Auger spectrum when the declination is restricted to a range from -15 to $24.8^{\circ}$. For declinations in -15 to $24.8^{\circ}$ range, the second break point in the TA spectrum occurs at $10^{19.59 \pm 0.06} \mathrm{eV}$ and for declinations from 24.8 to $90^{\circ}$, the second break point occurs at $10^{19.85 \pm 0.03} \mathrm{eV}$. These results are 


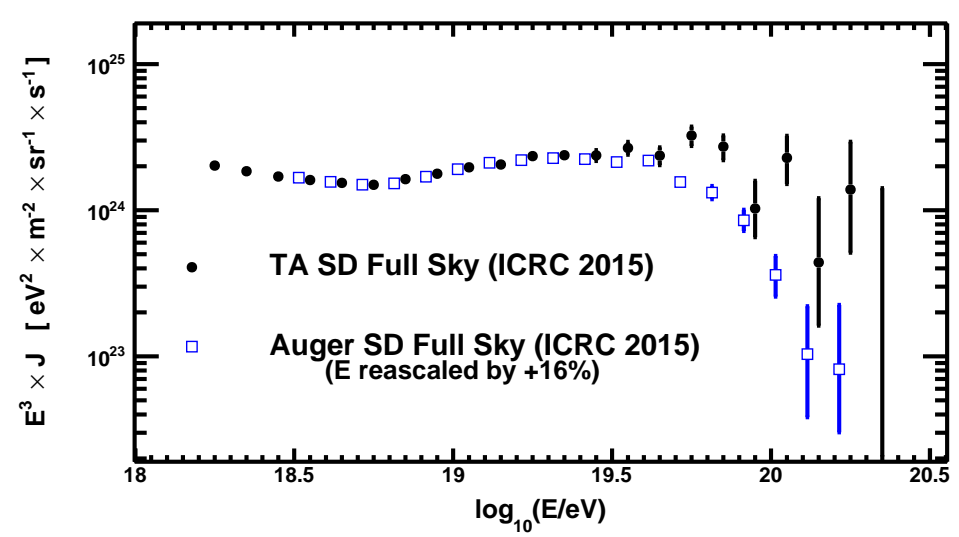

Figure 2: TA and Auger SD energy spectra. Auger energies have been rescaled by 1.16 to match the TA energy scale around the ankle region.

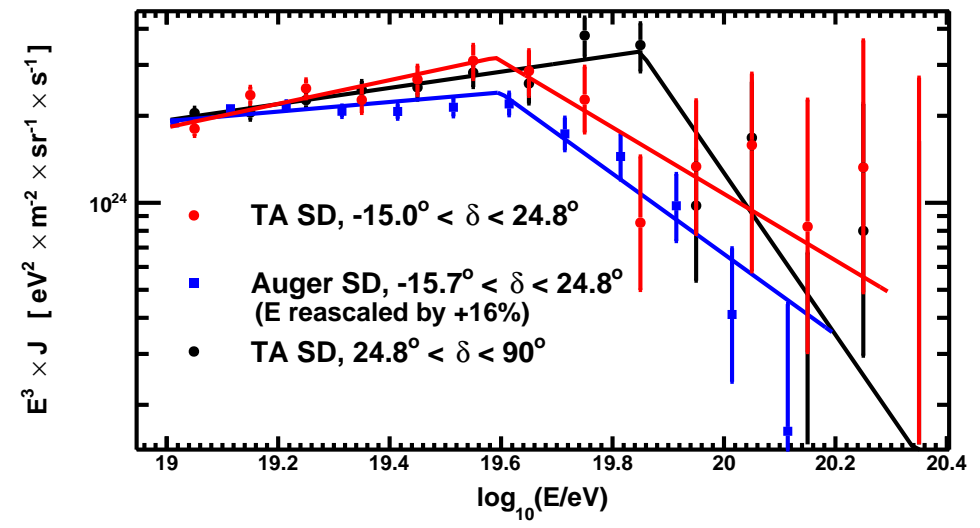

Figure 3: Comparison of Auger and TA SD energy spectra in different declination bands. Position of the second break point is different for events below and above the declination of $24.8^{\circ}$. We see a better agreement with the Auger when the TA and Auger [18] spectra are restricted to the declinations from -15 to $24.8^{\circ}$. Auger energies have been scaled up by 1.16 as before.

shown in Figure 3.

\section{Check of Systematic Uncertainties}

We first check whether the surface detector energy reconstruction has a bias that depends on the event energy or zenith angle. We check this by using events seen in common by the SD and FD and comparing the ratio of the SD energy to that of the FD for different slices in energy and zenith angle. As Figure 4 shows, no significant SD energy reconstruction biases are seen.

Above $10 \mathrm{EeV}$, the TA SD acceptance is independent of the event energy and the arrival direction (the aperture of the SD depends on the zenith angle only geometrically, as $\sin (\theta) \cos (\theta)$ ) [13]. 

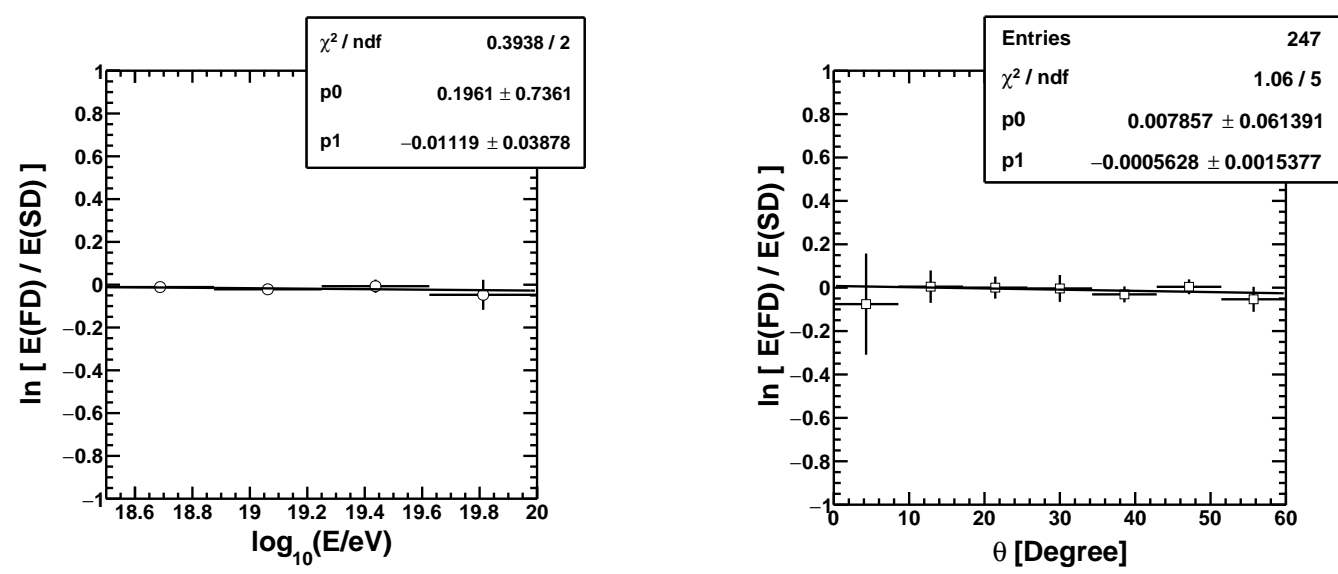

Figure 4: Check of the SD energy scale linearity. (Left panel) Ratio of the TA FD energy to the SD energy as a function of energy (Right panel) Ratio of the FD energy to the SD energy as a function of the event zenith angle $\theta$, for energies above $10 \mathrm{EeV}$. Linear fit is made to both figures, and the result is that the slopes are withing their fitting uncertainties in both figures, indicating that there are no significant energy reconstruction biases.

To verify this further, we perform the following test. We first note that cutting on the event declination below and above $24.8^{\circ}$ is equivalent to cutting on points inside and outside, respectively, of the $\theta, \phi$ constant declination contour shown in the left panel of Figure 4. $\theta$ is the zenith and $\phi$ is the azimuthal angles of the event arrival direction in the local sky. If we move this contour to the right in $\phi$ by $+90^{\circ}$, however (right panel of Figure 4), and consider two data sets which consist of events inside and outside of the contour, then the declination distributions of the two sets of data are roughly similar. If the spectrum difference is due to the declination only and not because of the acceptance or reconstruction biases in $\theta, \phi$, then the two spectra should be in a good agreement, as Figure 6 shows.

\section{Summary}

We have seen an evidence of the declination dependence of the second break point in the TA SD spectrum and we have demonstrated that this is not an instrumental effect. In the first 7 years of the TA SD data, the declination dependence of the second break point was a $3.9 \sigma$ effect. A preliminary analysis of the last 2 years of the TA SD data, from May 12, 2015 to May 11, 2017, showed a reduced statistical significance of the effect. The TA X 4 extension detector, which is currently being constructed, [19], is expected to provide additional data needed to measure the energy spectrum declination dependence more accurately.

\section{Acknowledgments}

The Telescope Array experiment is supported by the Japan Society for the Promotion of Science through Grants-in-Aid for Scientific Research on Specially Promoted Research (21000002) "Extreme Phenomena in the Universe Explored by Highest Energy Cosmic Rays" and for Scientific Research (19104006), and the Inter-University Research Program of the Institute for Cosmic Ray 

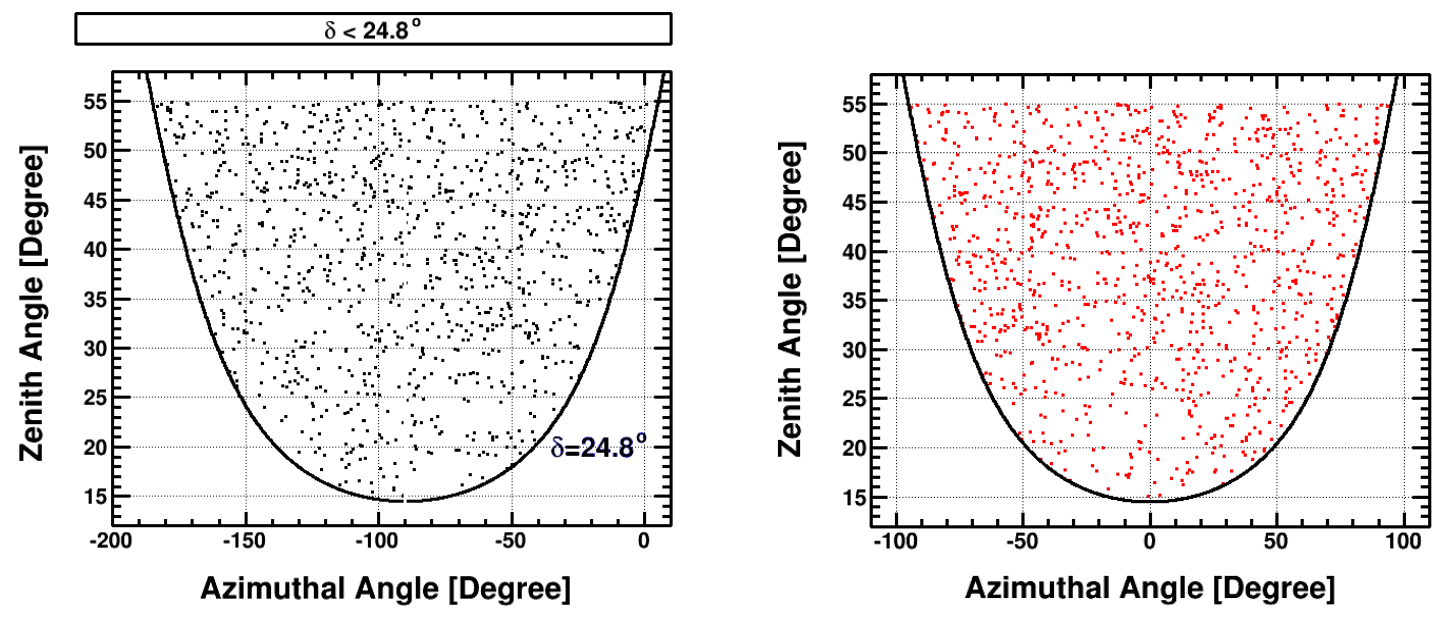

Figure 5: (Left panel) Cutting on declination above and below $24.8^{\circ}$ is equivalent to cutting on data below and above the solid curve, respectively. (Right panel): After moving the solid curve by $+90^{\circ}$ to the right, cutting on data below and above the solid line no longer corresponds to cutting on declination above and below $24.8^{\circ}$.
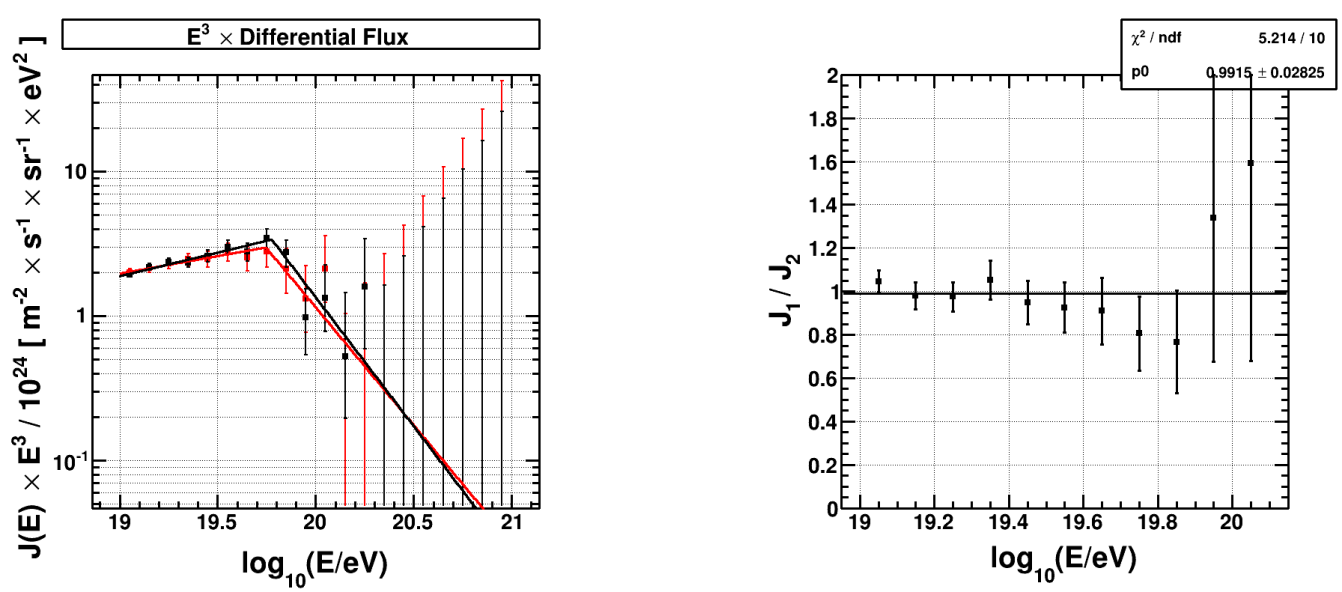

Figure 6: If the TA SD spectrum depends only on the declination then the spectra that correspond to the two data sets obtained by selecting events inside and outside of the $\theta$ vs $\phi$ curve in the left panel of the previous figure should be similar. Fluxes $J 1$ and $J 2$ that correspond to the two data sets are in good agreement (right panel), as the ratio $J 1 / J 2$ shows (left panel).

Research; by the U.S. National Science Foundation awards PHY-0601915, PHY-1404495, PHY1404502, and PHY-1607727; by the National Research Foundation of Korea (2015R1A2A1A01006870, 2015R1A2A1A15055344, 2016R1A5A1013277, 2007-0093860, 2016R1A2B4014967); by the Russian Academy of Sciences, RFBR grant 16-0200962a (INR), IISN project No. 4.4502.13, and Belgian Science Policy under IUAP VII/37 (ULB). The foundations of Dr. Ezekiel R. and Edna Wattis Dumke, Willard L. Eccles, and George S. and Dolores Doré Eccles all helped with generous donations. The State of Utah supported the project through its Economic Development Board, and the University of Utah through the Office of the 
Vice President for Research. The experimental site became available through the cooperation of the Utah School and Institutional Trust Lands Administration (SITLA), U.S. Bureau of Land Management (BLM), and the U.S. Air Force. We appreciate the assistance of the State of Utah and Fillmore offices of the BLM in crafting the Plan of Development for the site. Patrick Shea assisted the collaboration with valuable advice on a variety of topics. The people and the officials of Millard County, Utah have been a source of steadfast and warm support for our work which we greatly appreciate. We are indebted to the Millard County Road Department for their efforts to maintain and clear the roads which get us to our sites. We gratefully acknowledge the contribution from the technical staffs of our home institutions. An allocation of computer time from the Center for High Performance Computing at the University of Utah is gratefully acknowledged.

\section{References}

[1] R. U. Abbasi et al. [High Resolution Fly's Eye Collaboration], Phys. Rev. Lett. A 100 (2008) 101101

[2] A. Aab et al. [Pierre Auger Collaboration] JCAP 1508 (2015) 49

[3] T. Abu-Zayyad et al. [Telescope Array Collaboration], Astrophys. J. 768 (2013) L1 [arXiv:1205.5067 [astro-ph.HE]].

[4] V. Berezinsky et al., Phys. Rev. D 74 (2006) 043005.

[5] K. Greisen, Phys. Rev. Lett. 16 (1966) 183

[6] G. T. Zatsepin and V. A. Kuz'min, Sov. Phys. JETP Lett. 4 (1966) 114.

[7] R. U. Abbasi et al. [HiRes Collaboration], Phys. Rev. Lett. 104 (2010) 161101 [arXiv:0910.4184 [astro-ph.HE]].

[8] R. U. Abbasi, M. Abe et al. [Telescope Array Collaboration], Astropart. Phys. 64 (2014) 49 [arXiv:1408.1726 [astro-ph.HE]].

[9] A. Aab et al. [Pierre Auger Collaboration], Phys. Rev. D 90 (2014) 12, 122006 [arXiv:1409.5083 [astro-ph.HE]].

[10] T. Abu-Zayyad et al. [Telescope Array Collaboration], Nucl. Instrum. Meth. A 609 (2009) 227

[11] T. Abu-Zayyad et al. [Telescope Array Collaboration], Astropart. Phys. 39-40 (2012) 109 [arXiv:1202.5141 [astro-ph.IM]].

[12] T. Abu-Zayyad et al. [Telescope Array Collaboration], Nucl. Instrum. Meth. A 689 (2012) 87 [arXiv:1201.4964 [astro-ph.IM]].

[13] D. Ivanov, "Energy Spectrum Measured by the Telescope Array Surface Detector", doctoral thesis, Rutgers University, New Brunswick, New Jersey, October, 2012.

[14] J. Knapp and D. Heck, Nachr. Forsch. zentr. Karlsruhe 30 (1998) 27.

[15] S. Ostapchenko, Nucl. Phys. Proc. Suppl. 151 (2006) 143 [hep-ph/0412332].

[16] M. Kobal, Astropart. Phys. 259-273, 15 (2001)

[17] B. T. Stokes, R. Cady, D. Ivanov, J. N. Matthews and G. B. Thomson, Astropart. Phys. 35 (2012) 759 [arXiv:1104.3182 [astro-ph.IM]].

[18] I. Valiño, for the Pierre Auger Collaboration, in Proc. 34th ICRC 2015, The Hague, The Netherlands, PoS (ICRC2015) 271. 
[19] E. Kido [Telescope Array Collaboration], "The TAx4 experiment" Contribution ID CRI199, ICRC-2017, Bexco, Busan, Korea 